\title{
Advances in Mixing Technology: Recent Advances in Mixing Research and Development
}

\author{
Shunsuke Hashimoto, ${ }^{1}$ Naoto Ohmura, ${ }^{2}$ See-Jo Kim, ${ }^{3}$ and Shaliza B. Ibrahim ${ }^{4}$ \\ ${ }^{1}$ Division of Chemical Engineering, Graduate School of Engineering Science, Osaka University, 1-3 Machikaneyama, Toyonaka, \\ Osaka 560-8531, Japan \\ ${ }^{2}$ Department of Chemical Science and Engineering, Graduate School of Engineering, Kobe University, Rokkodai, Nada, \\ Kobe 657-8501, Japan \\ ${ }^{3}$ Department of Mechanical Design Engineering, Andong National University, Songchon-Dong, Kyungbuk, \\ Andong 760-749, Republic of Korea \\ ${ }^{4}$ Department of Civil Engineering, University of Malaya, 50603 Kuala Lumpur, Malaysia
}

Correspondence should be addressed to Shunsuke Hashimoto, shunsuke@cheng.es.osaka-u.ac.jp

Received 2 October 2012; Accepted 2 October 2012

Copyright (C) 2012 Shunsuke Hashimoto et al. This is an open access article distributed under the Creative Commons Attribution License, which permits unrestricted use, distribution, and reproduction in any medium, provided the original work is properly cited.

Mixing has been one of essential unit operations for chemical engineering processes. Among a number of mixers, stirred tanks that are available in a wide variety of tank sizes and impeller shapes are the most frequently adopted to homogenize different substances and to conduct chemical reactions in industrial chemical processes. In addition, there are various technologies for fluid mixing: static mixer, micromixer, unsteady agitation, eccentric agitation, and so on. Recently in various industrial processes, a wide range of operation for stirred tank is required depending on purposes and conditions, and high efficiency on mixing has been strongly required. In addition, the techniques of computer simulation analyses by the use of CFD software have been dramatically developed, which is essential to analyze the mixing mechanism. The main goal of this special issue was to gather contributions dealing with the latest breakthrough of mixing techniques. There is a collection of twelve papers in this special issue focused on mixing fundamental (2 papers), multiphase mixing (3 papers), chemical reactive mixing for metallurgical industry, and biotechnology (4 papers), and new technology of mixing (3 papers) ranging in topic from laminar-to-turbulent mixing by means of both experimental analyses and numerical simulations. The highlights of each paper are introduced as follows.

In the paper entitled by "Design mixers to minimize effects of erosion and corrosion erosion", E. E. Janz and J. Fasano from Chemineer, Inc. provide techniques for minimizing the wear on impellers cover the choice of impeller, size and speed of the impeller, alloy selection, and surface coating or coverings. An example is provided as well as an assessment of the approximate life improvement.

In the work entitled by "Effect of impeller agitation on preparation of tetra-n-butyl ammonium bromide semiclathrate hydrate slurries", S. Hashimoto et al. from Osaka University, which shows the effect of impeller agitation on particle size distribution and aggregation of tetra- $n$-butyl ammonium bromide (TBAB) semiclathrate hydrate slurries. The slurries containing TBAB solution and its semiclathrate hydrate have attracted a lot of interest as latent heat transport media. First of all, the effects of impeller rotational speed and impeller type on the particle size and frequency of aggregation are investigated. The results suggest that the particle size distribution and the frequency of particle aggregation are strongly affected by the intensity of shear rate and its uniformity, which is controllable with impeller type and its rotation speed.

In the research entitled by "Correlation of power consumption for several kinds of mixing impellers", Y. Kato et al. review the correlations of power consumption in nonbaffled and baffled agitated vessels with several kinds of impellers, which are developed in a wide range of Reynolds numbers from laminar-to-turbulent flow regions. The power correlations are based on Kamei and Hiraoka's expressions for paddle and pitched paddle impellers. The calculated correlation values 
agree well with experimental ones, and the correlations will develop the other types of impellers.

In the paper entitled by "Effect of temperature change on geometric structure of isolated mixing regions in stirred vessel", $\mathrm{N}$. Ohmura et al. show the effect of temperature change on the geometric structure of isolated mixing regions (IMRs) in a stirred vessel by the decolorization of fluorescent green dye by acid-base neutralization. The step temperature-change can dramatically reduce the elimination time of IMRs, as compared with a steady temperature operation. During the transient process from an initial state to disappearance of IMR, the IMR showed interesting three-dimensional geometrical changes, that is, simple torus with single filament, simple torus without filaments, a combination of crescent shape and circular tori, and doubly-entangled torus.

In the work entitled by "Phosphorus removal from wastewater using oven-dried alum sludge", S. A. Rashid and W. T. Mohammed deal with the removal of phosphorus from wastewater by using oven-dried alum sludge (ODS) as an adsorbent that was collected from Al-Qadisiya treatment plant (Iraq). The results show that the percent removal of phosphorus increases with the increase of oven dried alum sludge dose, but thr $\mathrm{pH}$ of solution has insignificant effect. In addition, the oven-dried alum sludge was effective in adsorbing phosphorus, and percent removal of phosphorus reaches $85 \%$ with increasing of contact time.

In the paper entitled by "Laminar mixing in stirred tank agitated by an impeller inclined", K. Takahashi et al. show the mixing performance in a vessel agitated by an impeller inclined itself, which is considered one of the typical ways to promote mixing performance by the spatial chaotic mixing. The effect of eccentric position of inclined impeller on mixing time was also studied and a significant reduction of mixing time is observed. To confirm the experimental results, the velocity profiles are calculated numerically and two novel numerical simulation methods are proposed.

In the research entitled by "Characterization of minimum impeller speed for suspension of solids in liquid at high solid concentration, using gamma ray densitometry", P. A. Tanguy et al. exhibit the limitations of applying conventional measurement techniques for the accurate characterization of critical impeller speed for just off-bottom suspension $(\mathrm{Njs})$ at high solid concentrations. Subsequently, the Gamma-Ray Densitometry technique for characterizing $\mathrm{Njs}$ is introduced, which can overcome the limitations of previous experimental techniques. The theoretical concept of this method is explained and experimental validation is presented to confirm the accuracy of the Gamma-Ray Densitometry technique. By utilizing the similarity to the incipient movement of solid particles in other systems, a theoretical model for $\mathrm{Njs}$ prediction is presented.

In the work entitled by "The effects of mixing, reaction rates and stoichiometry on yield for mixing sensitive reactions-Part I: model development", S. M. Kresta et al. provide the model and design of the effect of mixing, reaction rates, and stoichiometry on yield for mixing sensitive reactions: competitive-consecutive and competitiveparallel reactions. A detailed investigation of the effects of initial mixing condition (striation thickness), dimensionless reaction rate ratio and reaction stoichiometry on the yield of desired product shows that the stoichiometry has a considerable effect on yield. All three variables are found to interact strongly. Model results for 12 stoichiometries are used to determine the mixing scale and relative rate ratio, needed to achieve a specified yield for each reaction scheme. The results show that all three variables need to be considered when specifying reactors for mixing sensitive reactions. Incidentally, Professor S. K. also organizes another paper.

In the paper entitled by "Effect of mixing on microorganism growth in loop bioreactors", A. M. Al Taweel et al. show the impact of mixing on the promotion of microorganism growth rate using a multiphase forced circulation pipe-loop reactor model capable of identifying conditions under which it is possible to convert natural gas into single cell protein (SCP). The impact of mixing in the interphase mass transfer is found to exert a critical role in determining the overall productivity of the bioreactor, particularly at the high cell loadings needed to reduce the capital costs associated with the large-scale production needed for the production of relatively low-value SCP in a sustainable manner.

In the paper entitled by "Investigation of the pulsed annular gas jet for chemical reactor cleaning”, C. V. Fedorovich et al. provide the study of aerodynamic efficiency of the impulse system carried by numerical modeling and experimentally with the help of a specially created experimental facility. The characteristics of the pulsed jets mixing are investigated and designed pulsed peelings system has shown high efficiency and reliability in functioning that allows us to recommend it for wide spreading in chemical industry.

The research of S. Ibrahim entitled by "Zwietering's equation for the suspension of porous particles and the use of curved blade impellers" reveals $\mathrm{Njs}$ of porous palm shell activated carbon (PSAC) particles for a few impeller blades. Specific power per unit mass for all three impellers showed reduction towards minima as the concentration of particles increased. The result means that the Zwietering equation can be used to predict suspension for porous particles with adjustment to the solid particle density. $S$ values for curvedblade impellers are presented for the first time.

In the work entitled by "The effects of mixing, reaction rates and stoichiometry on yield for mixing sensitive reactions-part II: design protocols", S. M. Kresta et al. provide the design strategy of the effect of mixing, reaction rates, and stoichiometry on yield for mixing sensitive reactions.

\section{Acknowledgments}

The guest editors are also sincerely thankful to the editorial staff for the support, guidance, and efforts in welcoming this series of papers on a timely subject. We hope that this special issue will be available for the future innovation of mixing technology.

Shunsuke Hashimoto Naoto Ohmura See-Jo Kim

Shaliza B. Ibrahim 

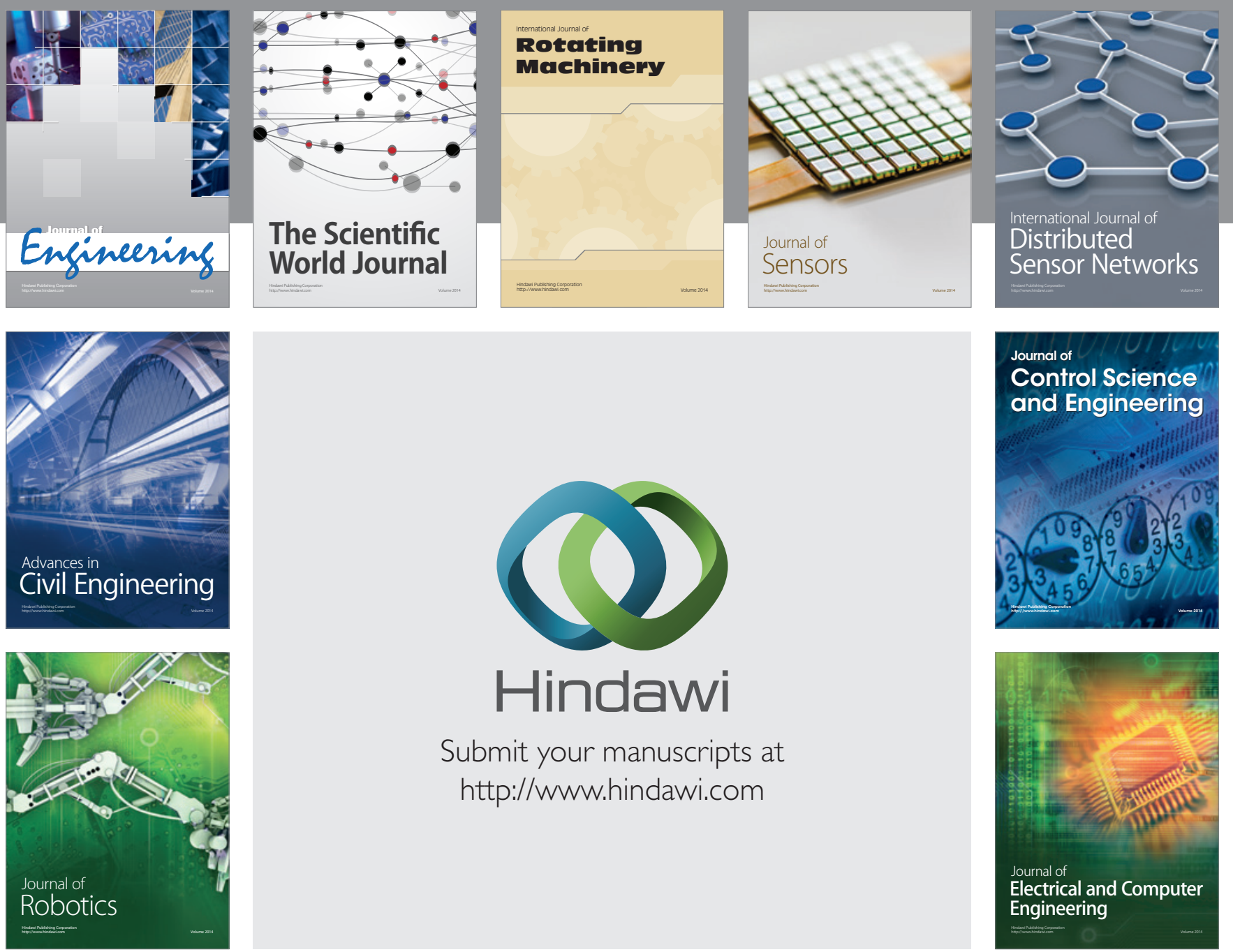

Submit your manuscripts at

http://www.hindawi.com
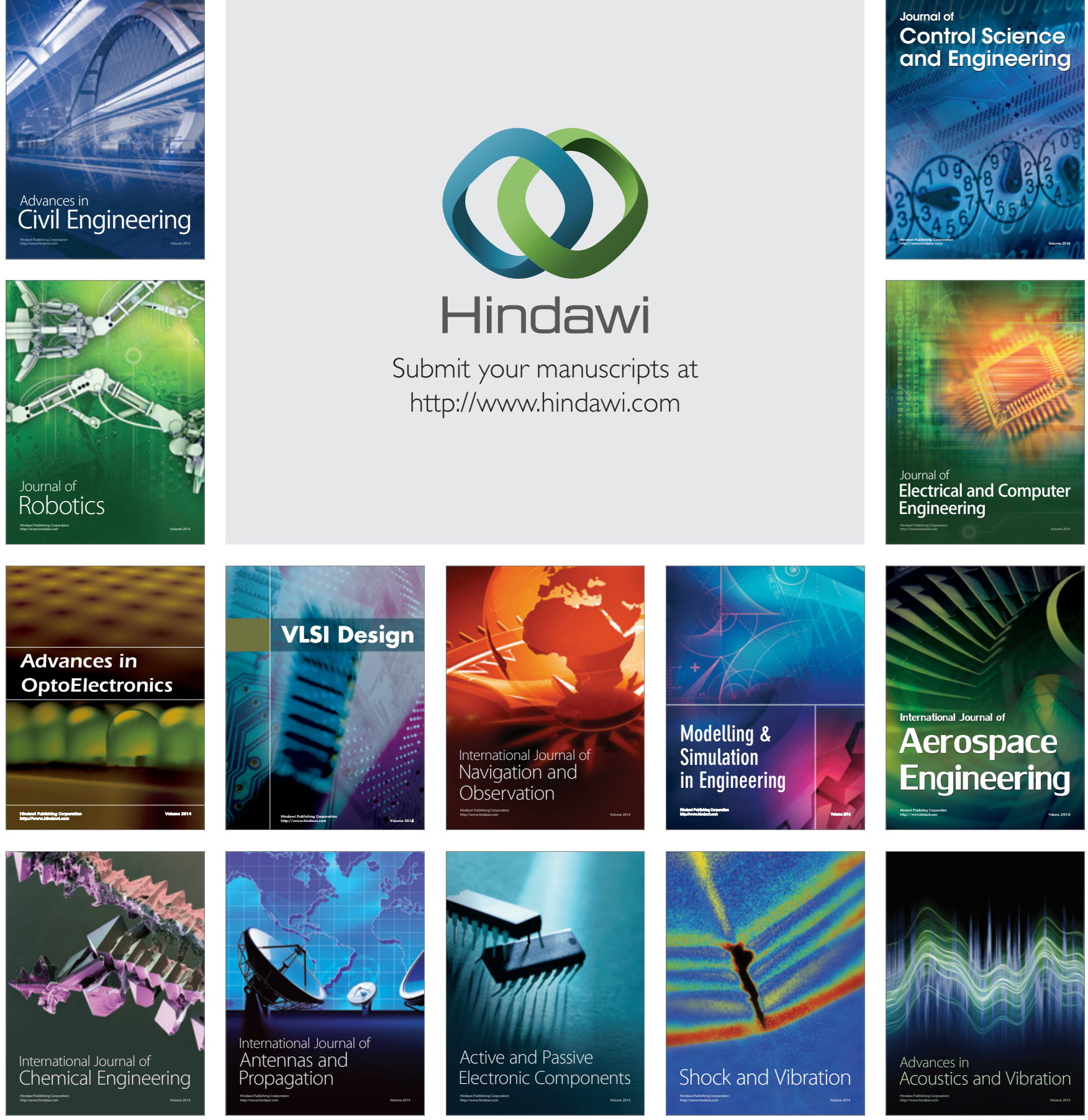